\title{
INTERFERENCE OF CORONAVIRUS INFECTION BY EXPRESSION OF IgG OR IgA VIRUS NEUTRALIZING ANTIBODIES
}

\author{
Isabel Sola, Joaquín Castilla, and Luis Enjuanes \\ Centro Nacional de Biotecnología \\ Consejo Superior de Investigaciones Científicas (CSIC) \\ Department of Molecular and Cell Biology \\ Campus Universidad Autónoma, Cantoblanco \\ 28049 Madrid, Spain
}

\section{ABSTRACT}

Mouse immunoglobulin gene fragments encoding the variable modules of the heavy (VH) and light (VL) chains of a transmissible gastroenteritis coronavirus (TGEV) neutralizing monoclonal antibody (MAb) have been cloned and sequenced. The selected MAb recognizes a highly conserved viral epitope and does not lead to the selection of neutralization escape mutants. Chimeric immunoglobulin genes with the variable modules from the murine MAb and constant modules of human gamma 1 and kappa chains were constructed using RT-PCR. These chimeric immunoglobulins were stably or transiently expressed in murine myelomas and COS cells, respectively. The secreted recombinant antibodies had radioimmunoassay (RIA) titers higher than $10^{3}$ and reduced the infectious virus more than $10^{4}$-fold. Recombinant dimeric IgA showed a 50 -fold enhanced neutralization of TGEV relative to a recombinant monomeric IgG1 which contained the identical antigen binding site. Epithelial cell lines stably-transformed with these constructs and expressing either recombinant IgG or IgA TGEV neutralizing antibodies reduced virus production by $>10^{5}$-fold after infection with homologous virus, although a residual level of virus production $\left(<10^{2} \mathrm{PFU} / \mathrm{ml}\right)$ remained in less than $0.1 \%$ of the cells.

\section{INTRODUCTION}

The mucosal immune system and its predominant effector, secretory immunoglobulin $\mathrm{A}(\operatorname{Ig} \mathrm{A})$, provide the initial immunologic barriers against most pathogens that invade 
the body at a mucosal surface (Mazanec et al., 1995; McGhee et al., 1989; Mestecky and $\mathrm{McGhee}, 1987)$. This is especially true for viruses, since resistance to infection has been strongly correlated with the presence of specific IgA antibody in mucosal secretions (Armstrong et al., 1990). Traditionally, the neutralization of viruses by immunoglobulins is thought to result from the binding of antibody to virion attachment proteins, thereby preventing adherence to epithelial cells surfaces. In addition, mucosal antibody interacts intracellularly with viruses preventing their replication, possibly by interfering with virus assembly (Mazanec et al., 1992).

New strategies, including the introduction of antibody genes into cells have been recently explored in model tissue culture systems. This approach may potentially be applied to in vivo protection of mucosal surfaces by gene therapy with antibody-encoding genes since monoclonal antibodies (MAbs) are now available against a vast range of viruses. Virus neutralizing MAbs may protect mucosal tissues against viral infections, however it is not known whether antibody secreting cells will provide protection to the neighboring tissues.

Transmissible gastroenteritis coronavirus (TGEV) infects both enteric and respiratory tissues and causes a mortality close to one hundred percent when newborn animals are infected (Enjuanes and Van der Zeijst, 1995). Full protection against TGEV can be provided by lactogenic immunity from immune sows (Saif and Wesley, 1992; Stone et al., 1977). Investigations by our laboratory into the mechanisms of TGEV neutralization (Suñé et al., 1990) and of antigenic and genetic variability (Sanchez et al., 1992; Sanchez et al., 1990) have led to the identification of a mouse MAb which neutralized all TGEV isolates tested, and also TGEV-related coronaviruses. No neutralization escape mutants (mar mutants) appeared when this MAb was employed (Gebauer et al., 1991).

We have studied the protection of epithelial cell monolayers against TGEV infection using expression plasmids encoding virus neutralizing MAbs. We found that cell lines stably-transformed with these MAb-producing vectors were substantially protected against TGEV challenge, however a small fraction of these cells continued to produce low levels of viral progeny.

\section{MATERIALS AND METHODS}

\subsection{Cell and Viruses}

Swine testis (ST) (McClurkin and Norman, 1966) and SV40 transformed monkey kidney COS-1 cells (ATCC CRL-1650), non secreting murine myeloma Sp2/0 (ATCC, CRL-1581), and MAb 6A.C3 and MAb 1G.A7-secreting murine hybridomas (Correa et al., 1988; Jiménez et al., 1986) were grown as described (Castilla et al., 1997). TGEV PUR46-MAD strain (Gebauer et al., 1991) was grown, purified, and titrated in ST cells as described (Jiménez et al., 1986). Vesicular stomatitis virus (VSV) was grown and titrated as described (Bullido et al., 1989).

\subsection{Immunofluorescence Microscopy}

$\mathrm{Sp} 2 / 0$ and ST cells expressing recombinant mouse-human (rMH) antibodies, recombinant mouse-swine (rMS) antibodies, and the MAb 6A.C3 secreting hybridoma were grown in microslide culture chambers (Miles Scientific). Immunofluorescence to detect rMAb expression was performed as described (Castilla et al., 1997). ST cells expressing 
rMH and rMS antibodies, or untransformed ST cells, were infected with TGEV. To detect recombinant antibodies and viral proteins in the same cells, double immunofluorescence was performed as previously described (Castilla et al., 1997).

\subsection{Radioimmunoassay (RIA), Virus Neutralization, and Western Blot Analysis}

The procedures for RIA, virus neutralization and Western blot have been described (Correa et al., 1988).

\subsection{RNA Extraction}

Total cytoplasmic RNA from hybridoma 6A.C3 was prepared as described (Castilla et al., 1997) PolyA ${ }^{+}$mRNA was isolated using the PolyATtract mRNA Isolation System (Promega).

\subsection{Sequencing, Characterization of 6A.C3 Variable (V)-Modules RNA, and Synthesis of MAb 6A.C3 cDNA Encoding the Variable Module}

MAb 6A.C3 polyA ${ }^{+}$mRNA was sequenced as previously described (Castilla et al., 1997).

\subsection{Construction of Immunoglobulin Expression Plasmids}

The expression plasmids pINLC6A and pINHC6A containing the recombinant light and heavy chains respectively were constructed as described (Castilla et al., 1997).

\subsection{Interference of TGEV Infection in ST Cell Lines Expressing Recombinant TGEV Neutralizing Antibodies}

Cloned and uncloned ST cell lines expressing recombinant TGEV neutralizing antibodies with $\mathrm{IgG}_{1}$ or IgA isotype were infected with TGEV as described (Castilla et al., 1997). Supernatants from infected and non-infected cells were analyzed at 1 to 6 days post infection (p.i.). Infections with vesicular stomatitis virus (VSV) were performed in parallel as a control.

\section{RESULTS}

\subsection{Sequence of Genes Encoding Variable Modules of a TGEV Neutralizing MAb}

In order to protect cell monolayers from virus infection using MAbs it is convenient to use an antibody with a high titer in virus neutralization, that recognizes an epitope present in all virus isolates, and which does not lead to the selection of neutralization escape mutants. These conditions were fulfilled by MAb 6A.C3 (Gebauer et al., 1991; Jiménez et al., 1986).

The sequence of MAb 6A.C3 V-modules was determined. The typical $\mathrm{L}$ and $\mathrm{H}$ chain organization was identified (data not shown). The sequences of MAb 6A.C3 L and H 
chain variable modules showed high homology with kappa (99.4\%) and gamma 1 (92.7\%) sequences of subgroup V and subgroup IIIC immunoglobulin genes, respectively. The J regions of the $\mathrm{L}$ and $\mathrm{H}$ chains belong to the $\mathrm{J}_{2}$ type.

\subsection{Generation of rMAbs 6A.C3}

Human $\kappa$ and $\gamma$ constant modules were flanked by the SV-40 early promoter and polyadenylation signals and were subcloned into plasmids pINLC6A and pINHC6A, respectively, which carry the mouse immunoglobulin enhancer at the 5' end of the expression cassettes. The engineering of a rIgA immunoglobulin with the same variable modules than the IIgG $_{1}$ has been described (Sola et al., 1997).

\subsection{Functional Analysis of Recombinant MAbs with Gamma 1 and Alpha Isotypes}

Western-blot analysis performed under non-reducing conditions demonstrated that MAb 6A.C3 with the IgG isotype was monomeric (molecular mass $150 \mathrm{kDa}$ ), while rIgA presented a dimeric structure of about $300 \mathrm{kDa}$ (results not shown). The secreted chimeric immunoglobulins expressed both in COS and SP $2 / 0$ cells bound TGEV by RIA with titers up to $10^{4}$, and neutralized virus infectivity around $10^{4}$-fold (neutralization indices, NIs, around 4) (Table 1).

The final aim of this work is to express the rMAbs in the swine enteric tract to examine their protective effect on mucosal surfaces against viral challenge. In these experiments IgA isotype antibodies are known to be more stable than those with an IgG isotype

Table 1. Functional characterization of recombinant antibodies

\begin{tabular}{|c|c|c|}
\hline \multirow[b]{2}{*}{ Antibody ${ }^{a}$} & \multicolumn{2}{|c|}{ Titer determined by } \\
\hline & $\mathrm{RIA}^{\mathrm{b}}$ & Neutralization index ${ }^{c}$ \\
\hline MAb-6A.C3 & $10^{4}$ & $>4$ \\
\hline rIgG-Sp $2 / 0$ cells & $10^{2}-10^{3}$ & $2-3$ \\
\hline rIgA-Sp $2 / 0$ cells & $10^{2}-10^{3}$ & 4 \\
\hline rIgG-COS cells & $10^{2}-10^{3}$ & 4 \\
\hline rIgA-COS cells & $10^{3}-10^{4}$ & 3 \\
\hline $\mathrm{r} \gamma$ & 5 & $<0.3$ \\
\hline \multicolumn{3}{|c|}{$\begin{array}{l}{ }^{\text {a }} \text { Chimeric immunoglobulins rIgGl and rIgA expressed in COS monkey } \\
\text { kidney cells and in Sp } 2 / 0 \text { myeloma cells were analyzed by RIA and vi- } \\
\text { rus neutralization assays. MAb- } 6 \mathrm{~A} . \mathrm{C} 3 \text {, antibody secreted by the original } \\
\text { hybridoma. rIgG-Sp } 2 / 0 \text { and rIgA-Sp } 2 / 0 \text {, recombinant mouse-human and } \\
\text { mouse-swine antibodies, respectively, secreted by transformed } \mathrm{Sp} 2 / 0 \\
\text { myeloma cells. rlgG-COS and rIgA-COS, recombinant mouse-human } \\
\text { and mouse-swine antibodies, respectively, secreted by transformed COS } \\
\text { cells. r } \gamma \text {, recombinant mouse-human gamma chain. }\end{array}$} \\
\hline \multicolumn{3}{|c|}{$\begin{array}{l}\text { bIA titer, highest dilution giving } 3 \text {-fold the background. Similar results } \\
\text { were obtained in more than five independent evaluations of the antibod- } \\
\text { ies secreted by the selected cell lines. }\end{array}$} \\
\hline \multicolumn{3}{|c|}{$\begin{array}{l}{ }^{c} \text { Neutralization index, } \log _{10} \text { of the ratio of the PFU after incubating the } \\
\text { virus in the presence of medium or the indicated MAb. Similar results } \\
\text { were obtained in the evaluation of more than five independent cultures } \\
\text { of transiently transformed COS cells. }\end{array}$} \\
\hline
\end{tabular}


(Lamm et al., 1995). To compare the neutralizing activity of $\operatorname{rgG}_{1}$ and $\operatorname{rg} A$, supernatants containing recombinant antibodies of the same RIA titer were used in neutralization assays. Recombinant IgA neutralized TGEV 50-fold more effectively than $\mathrm{rgG}_{1}$ as expected for a dimeric immunoglobulin.

\subsection{Generation of ST Cells Expressing rIgG $_{1}$ and $\operatorname{rIgA}$ and Evaluation of Their Resistance to TGEV Infection}

Porcine epithelial (ST) cells susceptible to infection with TGEV were transfected with DNA constructs encoding the chimeric $\mathrm{H}$ and $\mathrm{L}$ chains to produce either the $\mathrm{rIgG}_{1}$ or the rIgA TGEV specific antibodies. RIA titers of the recombinant MAbs in supernantants from the cell lines ranged from $10^{2}$ to $10^{3}$. Immunofluorescence microscopy studies using immunoglobulin-specific antibodies revealed that, before the stably transformed cells were cloned, 10 to $15 \%$ of the cells of each line expressed the recombinant MAbs (results not shown). Cell lines expressing $\mathrm{rIgG}_{1}$ or rIgA TGEV-specific antibodies were infected with TGEV. Transformed cell lines were apparently resistant to TGEV infection (Figure 1A) since no cytopathic effect was observed $48 \mathrm{~h}$ post-infection while untransformed ST cell monolayers were completely lysed. TGEV-infected ST cells produced $10^{7} \mathrm{PFU} / \mathrm{ml}$ while IgG1 and IgA-transformed ST cell supernatants had titers which dropped to $10^{3}$ $\mathrm{PFU} / \mathrm{ml}$ (Figure 1B). This inhibition in viral synthesis was specific, since VSV grew to the same titer in transformed and untransformed cells (Figure 1B). While there was a significant $\left(10^{4}\right.$-fold $)$ reduction in virus synthesis in the antibody-producing cells, they were not fully resistant to TGEV infection since a residual level of virus synthesis $\left(10^{3} \mathrm{PFU} / \mathrm{ml}\right)$ persisted in the absence of any discernible cytopathic effect.

\subsection{Interference of TGEV Infection in Cloned ST Cell Lines Expressing Recombinant Antibodies}

To determine whether the residual infection of transformed ST cell lines by TGEV was due to the presence of a large proportion of cells that did not produce the antibody, two cell lines producing the highest levels of either $\operatorname{rgG}_{1}$ or $\mathrm{rIgA}$ antibodies were cloned by limiting dilution and infected. All cloned cells expressed the expected rMAbs, either $\mathrm{rIgG}_{1}$ or $\mathrm{rIgA}$ as determined by immunofluorescence microscopy (Figure 2a and 2c) and $>99.9 \%$ were free of viral antigens (Figure $2 b$ and $2 \mathrm{~d}$ ). No cytopathic effect was observed at $72 \mathrm{~h} \mathrm{p}$.i. in the transformed cells, in contrast to the complete lysis observed in the untransformed ST cell monolayer, similarly to what was observed (Figure 1) in the uncloned cells (results not shown).

Next, the kinetics of antibody production, viral synthesis, and cytopathic effects were determined (Figure 3). Virus production in the supernatant of transformed cultures was reduced from approximately $10^{7.5} \mathrm{PFU} / \mathrm{ml}$ to $<10^{2} \mathrm{PFU} / \mathrm{ml}$. Cytopathic effects were not detected in rIgG-producing cells (Figure 3C) and only minor effects were observed at $72 \mathrm{~h}$ p.i. in rIgA-producing cells. A high reduction in the virus titer was detected $\left(>10^{5}-\right.$ fold) but a residual infectivity $\left(<10^{2} \mathrm{PFU} / \mathrm{ml}\right)$ persisted in the supernatants. Low levels of infectious virus particles were produced by the transformed ST cells in the presence of virus neutralizing antibody (Figure $3 \mathrm{~A}$ and $3 \mathrm{~B}$ ). To test whether a neutralization resistant virus had been selected, the virus produced at 1, 2, 3, and $6 \mathrm{~d}$ p.i. was studied. Neutralization assays by different MAbs demonstrated that a neutralization escape mutant was not present. 
A

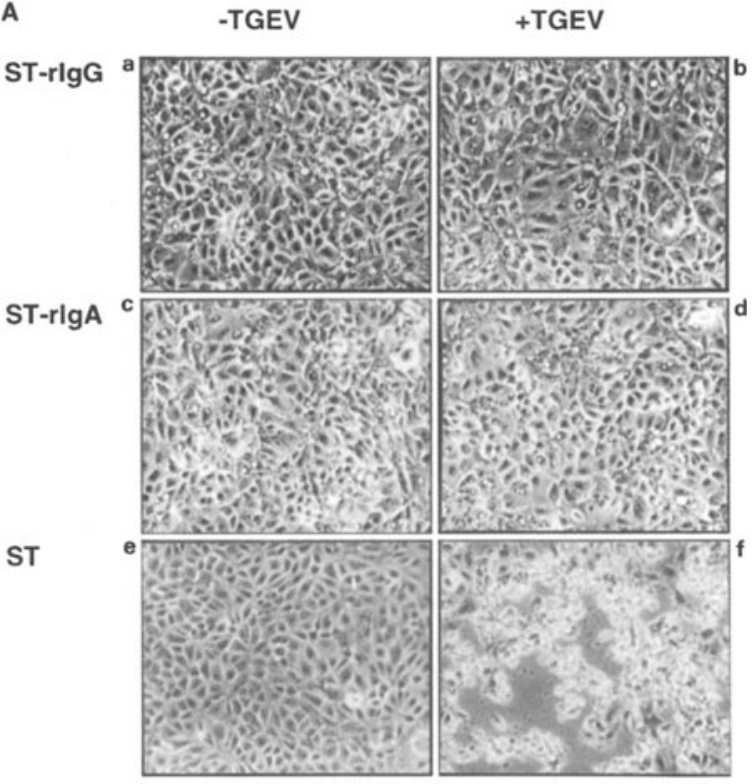

B

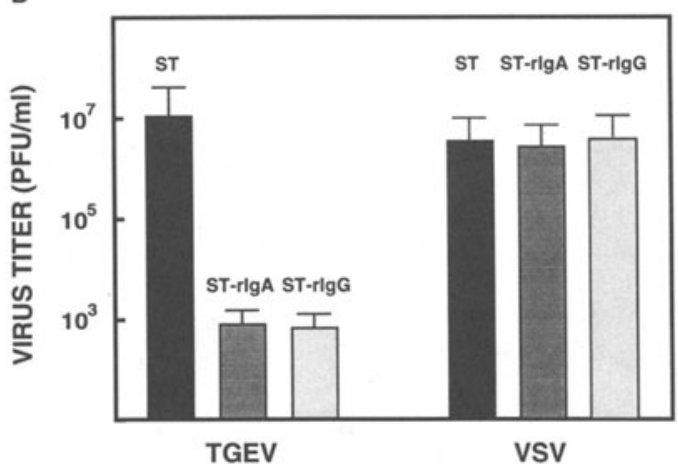

Figure 1. Resistance of rMAb producing ST cells to virus infection. (A) Phase contrast microscopy of rIgG1 $(\mathrm{a}, \mathrm{b})$ or rlgA (c, d) producing ST cells, or of untransformed cells (e, f). Cells were infected with TGEV (MOI 0.5) (b, d, f) or uninfected (a, c, e). (B) Specificity of the virus growth inhibition in antibody producing cell lines. Untransformed ST cells or ST cells producing TGEV neutralizing rIgA or IgG1 antibodies derived from MAb 6A.C3 were infected with TGEV or VSV. Supernatants were harvested $48 \mathrm{~h}$ p.i. and titrated on ST cells. Thin vertical bars, standard error. ST, untransformed cells. ST-rIgG and ST-rIgA, cells producing IIgG or rIgA neutralizing TGEV, respectively. The mean \pm the standard deviation of three experiments is shown. 
Figure 2. Immunofluorescence of TGEV-infected ST cells expressing neutralizing MAbs. Control ST cells or cells expressing MAbs were infected with TGEV. At $48 \mathrm{~h}$ p.i. cells were fixed with methanol and stained by two color immunofluorescence to detect antibodies and viral proteins. Recombinant antibodies were detected in transformed ST cells (a and c) but not in untransformed cells (e). Conversely, viral antigens were detected in untransformed cells (f) but not $(<0.1 \%$ of cells) in rIgG (b) or rIgA (d) transformed cells. ST, untransformed cells. ST-rIgG and STrIgA, cells producing rlgG or $\operatorname{rlgA}$ neutralizing TGEV, respectively. Anti-MAb and anti-TGEV, cells stainedby immunofluorescence using antibodies specific for immunoglobulins or TGEV.

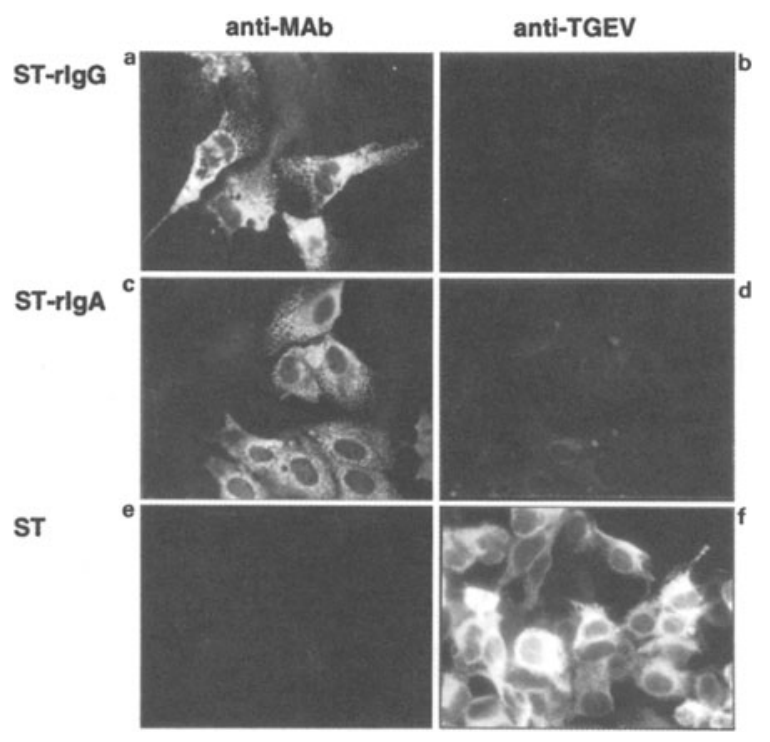

\section{DISCUSSION}

The genetic engineering and the anti-viral efficacy of two TGEV neutralizing MAbs with the same binding site and $\operatorname{IgG} 1$ and $\operatorname{IgA}$ isotypes are described. The dimeric $\mathrm{rMAb}$ with an $\operatorname{IgA}$ isotype had a 50 -fold higher efficiency in virus neutralization assays than the monomeric IgG1 isotype. Transformation of ST cells with plasmids encoding TGEV-neutralizing rMAbs with $\operatorname{IgG}_{1}$ or $\operatorname{IgA}$ isotype reduced virus infectivity $>10^{5}$-fold and prevented the appearance of cytopathic effects in vitro. A low level of virus production was detected in a few antibody producing cells.

A high level of functional chimeric antibody was produced in immunoglobulin-gene transiently-transformed COS cells and in stably-transformed myeloma cell lines ( $\mathrm{Sp} 2 / 0)$ as were the epithelial (ST) cells. This indicates that in principle, these DNA constructs could be used to transform other epithelial cells such as those found at porcine mucosal cell surfaces making them prime candidates for use as a somatic cell gene therapy-based vaccination strategy.

A highly significant $\left(>10^{5}\right.$-fold) reduction in the virus recovery in rIgG- or rIgA-producing cell lines has been demonstrated. This reduction in virus production in conjunction with other natural antiviral mechanisms including non-specific immune effector molecules such as interferon may be sufficient to eliminate the residual virus or the virus producing cells to provide protection against virus infection in vivo.

ST cell lines expressing a TGEV-neutralizing rMAb, in which only 10 to $15 \%$ of the cells were transformed, were largely protected against TGEV infection. Nevertheless, protection was not complete. Supernatants of transformed ST cell clones had virus titers $<10^{2}$ $\mathrm{PFU} / \mathrm{ml}$, very low in comparison with the $10^{7} \mathrm{PFU} / \mathrm{ml}$ observed in untransformed cells under the same conditions. The decrease in virus titer probably was not due to a non-specific reduction in the capacity of the transformed cells to produce virus, since the transformed 
A

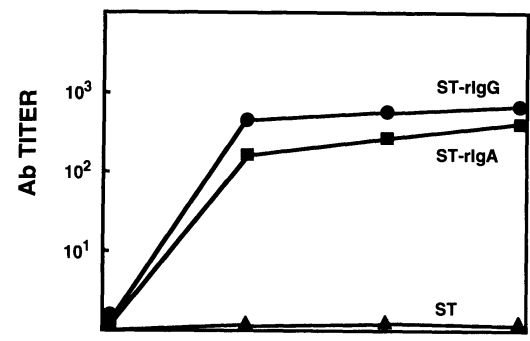

B

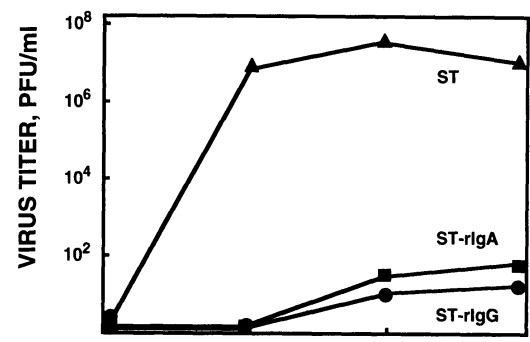

C

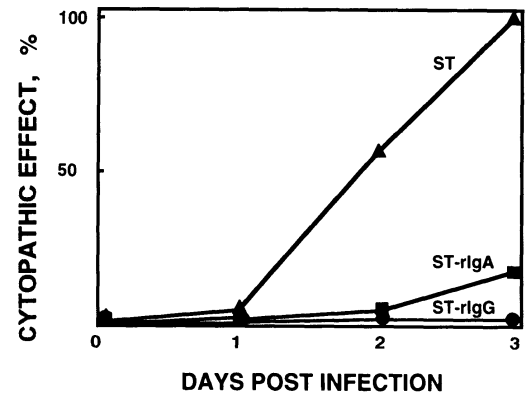

Figure 3. Kinetics of antibody production, virus titer, and cytopathic effects in recombinant antibody producing cells. Untransformed ST cells or cell lines producing recombinant IgG1 or IgA were infected with TGEV. (A) Antibody titers for TGEV were determined by RIA. (B) Virus titers were determined using ST cells. (C) The cytopathic effect was calculated by counting the cells that remained bound to culture plates and was expressed as the percentage of the cells that were detached. ST, untransformed cells. ST-rIgG and ST-rIgA, cells producing rIgG or rIgA neutralizing TGEV, respectively. One representative experiment selected from three assays with similar results is shown.

ST cells produced VSV as efficiently as did the untransformed cells. In addition, the TGEV titer was similar in the supernatant of cell lines in which only 10 to $15 \%$ of the cells were transformed, than in the cloned transformed cell lines in which $100 \%$ of the cells were antibody producers. The most likely explanation for this residual virus persistence is that the antibody-virus neutralization complex is reversible and, at the antibody concentrations present in the supernatants of the rMAb transformed cells, a small virus fraction $\left(10^{2}-10^{3} \mathrm{PFU}\right)$ remains as free infectious virus, while most of the virus $\left(10^{4}\right.$ to $10^{5}$ PFU) is in the form of non-infections virus-antibody complexes (Suñé et al., 1990).

Extracellular neutralization is likely to be responsible for the results of this study, because the antibody is continuously released into the medium, even during virus infection, and in the immunoglobulin gene-transformed uncloned cells about 85 to $90 \%$ of the cells did not produce the antibody yet they were still protected from TGEV infection. Intracellular neutralization of virus in cultured cells has been demonstrated in the influenza and Sendai virus systems during transcytosis of dimeric IgA (dIgA) (Mazanec et al., 1995). This mechanism may also contribute part of the observed protection in antibodyproducing ST cells infected with TGEV since during virus and antibody synthesis these 
proteins could co-localize leading to an intracellular interference of protein transport as described in other systems (Mazanec et al., 1992).

A neutralization escape mutant was not selected for because the virus produced at 1 and 6 days p.i. was neutralized with the same efficiency by MAb 6A.C3, produced by the transformed ST cells, and by MAb 1G.A7, which binds to a different antigenic subsite of the TGEV spike protein (Correa et al., 1988; Gebauer et al., 1991). This is in agreement with our previous results indicating that the epitope recognized by MAb 6A.C3 is an interspecies-conserved epitope which seems to be essential for virus replication, and for which neutralization escape mutants have never been observed (Sanchez et al., 1990).

The transient expression of virus neutralizing antibodies in mucosal surfaces could be used to provide immediate protection of these tissues against viral infection. This type of somatic cell gene therapy may particularly be efficacious to protect newborn animals. This new vaccination strategy may also be advantageous both in the prevention of virus infections, and therapeutically, following the possible ingestion of a variety of viruses, where fast immune intervention is required in a defined tissue, since viral vectors could express antibody genes within two to three hours following delivery.

\section{ACKNOWLEDGMENTS}

This work has been supported by grants from the Consejo Superior de Investigaciones Científicas, the Comisión Interministerial de Ciencia y Tecnología (CICYT), La Consejería de Educación y Cultura de la Comunidad de Madrid, and Laboratorios Sobrino (Cyanamid Group) from Spain, and the European Communities (Projects Biotechnology and FAIR). JC and IS received fellowships from the Department of Education, University and Research of the Gobierno Vasco and the Consejo Superior de Investigaciones Científicas (Spain), respectively.

\section{REFERENCES}

Armstrong, S. J., Outlaw, M. C., and Dimmock, N. J., 1990, Morphological studies of neutralization of influenza virus by IgM, J. Gen. Virol. 71: 2313-2319.

Bullido, M. J., Correa, I., Jiménez, G., Suñé, C., Gebauer, F., and Enjuanes, L., 1989, Induction of transmissible gastroenteritis coronavirus-neutralizing antibodies in vitro by virus-specific $\mathrm{T}$ helper cell hybridomas, $J$. Gen. Virol. 70: $659-672$.

Castilla, J., Sola, I., and Enjuanes, L., 1997, Interference of coronavirus infection by expression of immunoglobulin $\mathrm{G}(\mathrm{IgG})$ or IgA virus-neutralizing antibodies, J. Virol. 71: 5251-5258.

Correa, I., Jiménez, G., Suñé, C., Bullido, M. J., and Enjuanes, L., 1988, Antigenic structure of the E2 glycoprotein from transmissible gastroenteritis coronavirus, Virus. Res. 10: 77-94.

Enjuanes, L., and Van der Zeijst, B. A. M., 1995, Molecular basis of transmissible gastroenteritis coronavirus epidemiology, in: The Coronaviridae (S. G. Siddell, eds.), Plenum Press, New York, pp. 337-376.

Gebauer, F., Posthumus, W. A. P., Correa, I., Suñé, C., Sánchez, C. M., Smerdou, C., Lenstra, J. A., Meloen, R., and Enjuanes, L., 1991, Residues involved in the formation of the antigenic sites of the $S$ protein of transmissible gastroenteritis coronavirus, Virology 183: 225-238.

Jiménez, G., Correa, I., Melgosa, M. P., Bullido, M. J., and Enjuanes, L., 1986, Critical epitopes in transmissible gastroenteritis virus neutralization, J. Virol. 60: 131-139.

Marasco, W. A., Haseltine, W. A., and Chen, S., 1993, Design, intracellular expression, and activity of a human anti-human immunodeficiency virus type 1 gp120 single-chain antibody, Proc. Natl. Acad. Sci. USA 90: 7889-7893.

Mazanec, M. B., Coudret, C. L., and Fletcher, D. R., 1995, Intracellular neutralization of influenza virus by immunoglobulin A anti-hemagglutinin monoclonal antibodies, J. Virol. 69: 1339-1343. 
Mazanec, M. B., Kaetzel, C. S., Lamm, M. E., Fletcher, D., and Nedrud, J. G., 1992, Intracellular neutralization of virus by immunoglobulin A antibodies, Proc. Natl. Acad. Sci. USA 89: 6901-6905.

McClurkin, A. W., and Norman, J. O., 1966, Studies on transmissible gastroenteritis of swine. II. Selected characteristics of a cytopathogenic virus common to five isolates from transmissible gastroenteritis, Can. J. Comp. Vet. Sci. 30: 190-198.

McGhee, J. R., Mestecky, J., Elson, C. O., and Kiyono, H., 1989, Regulation of IgA synthesis and immune response by T cells and interleukins, J. Clin. Immunol. 9: 175-199.

Mestecky, J., and McGhee, J. R., 1987, Immunoglobulin A (IgA): molecular and cellular interactions involved in IgA biosynthesis and immune response, Adv. Immunol. 40: 153-245

Saif, L. J., and Wesley, R. D., 1992, Transmissible gastroenteritis, in: Diseases of Swine, (A. D. Leman, B. E. Straw, W. L. Mengeling, S. D’Allaire, and D. J. Taylor, eds.), Wolfe Publishing Ltd, Ames. Iowa, pp. 362-386.

Sanchez, C. M., Gebauer, F., Suñé, C., Méndez, A., Dopazo, J., and Enjuanes, L., 1992, Genetic evolution and tropism of transmissible gastroenteritis coronaviruses, Virology 190: 92-105.

Sanchez, C. M., Jiménez, G., Laviada, M. D., Correa, I., Suñé, C., Bullido, M. J., Gebauer, F., Smerdou, C., Callebaut, P., Escribano, J. M., and Enjuanes, L., 1990, Antigenic homology among coronaviruses related to transmissible gastroenteritis virus, Virology 174: 410-417.

Sola, I., Castilla, J., Pintado, B., Sánchez-Morgado, J. M., Whitelaw, B., Clark, J., and Enjuanes, L., 1998, Transgenic mice secreting coronavirus neutralizing antibodies into the milk. J. Virol. 72:3762-3772.

Stone, S. S., Kemeny, L. J., Woods, R. D., and Jensen, M. T., 1977, Efficacy of isolated colostral IgA, IgG, and $\operatorname{IgM}(\mathrm{A})$ to protect neonatal pigs against the coronavirus transmissible gastroenteritis, Am. J. Vet. Res. 38: $1285-1288$.

Suñé, C., Jiménez, G., Correa, I., Bullido, M. J., Gebauer, F., Smerdou, C., and Enjuanes, L., 1990, Mechanisms of transmissible gastroenteritis coronavirus neutralization, Virology 177: 559-569. 